\title{
PROPOSAL
}

\section{A Muon Exposure in the Tohoku High Resolution Bubble Chamber}

\author{
Brown-FNAL-Indiana-IHEP-MIT-Tohoku-Tohoku Gakuin \\ Collaboration
}

Brown University, Providence, Rhode Island 02912

A. Chen, A. Shapiro, M. Widgoff

Fermilab

S. Childress, T. Murphy

Indiana University, Bloomington, Indiana 47401

E.D. Alyea, Jr.

Institute for High Energy Physics (IHEP), Beijing, PRC

C. Mao, Y. Tai, S. Wang, Y. Wu, S.W. Xu, C. Zhao, L. Mu

Massachusetts Institute of Technology, Cambridge, Massachusetts 02139

E.S. Hafen, P. Haridas, C. Milstene, S.H. Oh, I.A. Pless

Tohoku University, Sendai, Japan

T. Kitagaki, S. Tanaka, H. Yuta, A. Yamaguchi, K. Abe, K. Hasegawa, Y. Morita, K. Furuno, M. Sasaki, T. Akagi, H. Suzuki, H. Masuda

Tohoku Gakuin University, Tagajyo, Japan

M. Higuchi, M. Sato and Y. Hoshi 


\section{A MUON EXPOSURE IN THE TOHOKU HIGH RESOLUTION BUBBLE CHAMBER}

\section{Introduction}

The Tohoku Bubble Chamber Hybrid System consists of the Tohoku 1.4m high resolution freon bubble chamber and a downstream muon detection system. The system is located at Lab-F and being used for a high energy neutrino experiment, E745.

The E745 collaboration proposes here to expose the Tohoku Bubble Chamber Hybrid System to muon beam of about $300 \mathrm{GeV} / \mathrm{c}$. This experiment will make possible mutual calibrations and comparisons between the muon data and the neutrino results of E745 in the same detector at comparable energies.

The advantages and disadvantages of a bubble chamber experiment are complementary with those of electronic experiments. The $4 \pi$ detection of a bubble chamber produces good systematics, and its high spatial resolution makes possible the direct observation of strange and charm particles. However, the statistics is quite low for leptonic interactions in a bubble chamber experiment. There has been, for this reason, only one muon bubble chamber experiment, the $16 \mathrm{GeV} / \mathrm{c} \mu$

exposure in the SLAC 40 inch $\mathrm{BC}^{1}$ ). However, the $\mathrm{E745}$ bubble chamber system has shown its ability to execute a high energy neutrino experiment (for example Fig. 3a,b,c,d), and we now propose a unique bubble chamber experiment for high energy muons.

Most of the members of the E745 collaboration were previously involved in the SLAC experiment BC72/73/75 which was a study of the photoproduction of charm, 
strangeness, and vector mesons by $20 \mathrm{GeV}$ photons in the 40 inch bubble chamber ${ }^{2)}$. This proposed experiment is a natural extension from the point of view of photon interactions for the collaboration.

\section{Physics Aims}

A. In the deep inelastic region, the muon produced interaction data are directly comparable with the neutrino interaction results in approximately the same energy region, and we are using the same detector. The kinematics is the same in both interactions, and the intermediate quark states are very similar, Fig. 1. Charged lepton induced events have additional information about the incoming particles compared with neutral lepton events. Therefore this experiment will be a good calibration for E745. From the opposite point of view, the analysis method that was applied to neutral lepton events will give additional information for the analysis of charged lepton events, in addition to simultaneously measured muon scattering data. This will be a mutual calibration between bubble chamber and electronic scattering experiments. It is noted that the data from this experiment will be good in its systematics. Fig. 2 (a)-(c) show the distributions of $Q^{2}, \nu$ and $W$ obtained in E745 1985 run. The scale under (c) shows the $W_{\max }$ for each $P_{\mu^{\cdot}} A \sim 300 \mathrm{GeV} / \mathrm{c}$ muon beam will yield data which can be compared with the $\mathrm{E745}$ data.

B. This experiment will cover a wide range of $Q^{2}, Q^{2} \geq 0$ because of the triggering method mentioned later. Production of vector mesons, strange particles and charm particles will be studied over the whole range from the hadron-like to point-like regions of virtual photons. Direct observation of decays in space will give us a clean sample of $V^{\circ}$ and charm particles. Approximately $2800 \mathrm{~V}^{\circ}$ and 150 charm particles will be detected and investigated in each $Q^{2}$ region. There have been extensive indirect studies ${ }^{3}$ ) 
these processes but ours are direct. Production of meson-baryon pairs in strange and charm events will also be an interesting subject.

C. The study of charm baryons

The high resolution of the bubble chamber, and the identification capability for e and $\mu$ will make possible a study of charmed baryons.

D. Nuclear effect

In E545, a 350 GeV $\nu$-d experiment, we have studied the $\mathrm{R}=(\mathrm{d} \sigma / \mathrm{dx})_{\mathrm{Fe}} /(\mathrm{d} \sigma / \mathrm{dx}) \mathrm{d}$ by a comparison of events in the bubble chamber liquid and bubble chamber wall ${ }^{4}$ ). The same study will be made in the E745 1987 run for a higher energy region. A similar study will be carried out in this muon experiment. It includes the nuclear effect in $\mathrm{V}^{\circ}$ production. The number of usable events occurring in the chamber wall will be about a quarter of the events in the liquid.

Beam

A typical beam design is as follows

Beam: muon

Momentum: $\quad \sim 300 \mathrm{GeV}$, known momentum

Mode: $\quad$ pulsed. width 1 msec at base

Ping timing: every 4 seconds, six pings in a 20 second flat top

These figures can be modified to fit actual beam line condition.

Concerning track density in the bubble chamber, the SLAC $40^{\circ}$ bubble chamber experiment ${ }^{1)}$ accepted about $100 \mu$ 's in a region $1.2 \mathrm{~cm}(\mathrm{w}) \times 11 \mathrm{~cm}(\mathrm{~h})$. In this experiment about $500 \mu$ 's/pulse will give a similar projected density of beam tracks in the normal pictures. The maximum tolerable flux of muons will be determined by a halo muon beam test during the 1987 E745 run. 


\section{Detector}

The basic system is the same as that of the E745 1987 run. Figure 4 shows the 1.4 meter Tohoku freon bubble chamber + downstream muon detection system.

A. Bubble chamber optics

The six lens normal optics and two eye holographic optics are as used in the E745 1987 run. Normal pictures are taken every expansion of the chamber; a total of 662,000 pictures record all events occurring in the bubble chamber. The holographic pictures, about a third of the number of normal pictures, are initiated by the trigger system. Measurement of the holographic image is only made on events selected in the normal pictures.

B. Muon detection

Slow muons below $5 \mathrm{GeV} / \mathrm{c}$ are analyzed in the bubble chamber and identified by an iron absorber. Fast muons are analyzed and identified by the muon magnet (19 KG $\times 205 \mathrm{~cm})$. The muon tracking is accomplished by drift chamber, PWCs and limited streamer tube planes.

C. Triggering

Since the triggering is important in a muon experiment, we will add an upstream beam ID hodoscope system, EM-calorimeter (electromagnetic absorber), hadron calorimeter (hadron identifier), and a downstream muon identifier. The scintillation hadron calorimeter is the most essential element in the triggering. It is a W-(or $\nu$-) trigger instead of standard scattering angle trigger. It will function for the whole range of $Q^{2}, Q^{2} \geqslant Q_{\min }$, Fig. 5. Since a broad muon beam, $30 \mathrm{~cm}(\mathrm{w}) \times 60 \mathrm{~cm}(\mathrm{~h})$ is proposed, a scattering angle trigger is not practical. The electromagnetic absorber suppresses electromagnetic showers from the bubble chamber region. Hadronic events are then identified by the hadron calorimeter. In order to suppress electromagnetic events occurring in the EM- 
calorimeter or hadron calorimeter, a "single veto" at A, B and C in Fig. 6 is used, which rejects an event if any of six elements reports a single passing track. The upstream beam ID and downstream muon ID signals are combined. The optimum parameters for the hadron calorimeter will be decided by making a test during the E745 1987 run.

Final selection of events is done by analyzing the bubble chamber pictures. The role of the trigger in this experiment is to reduce the loss of the inelastic events.

\section{Events}

We expect $662 \mathrm{~K}$ expansions of the bubble chamber in the 6 ping operation mode $(6$ per $\mathrm{min}) \times(60 \times 24 \mathrm{hr} \times 30 \mathrm{~d} \times 5 \mathrm{mo}) \times .71 \times 0.80 \times 0.90=662 \mathrm{~K}$ expansions.

Accelerator operation: 10 days $/ 2$ weeks $=0.71$,

Beam efficiency $=0.80$,

Bubble chamber efficiency $=0.90$

With a normal picture rate of one every expansion and an average $500 \mathrm{muon} / \mathrm{ping}$, the total flux $\mathrm{N}_{\mu}=3.3 \times 10^{8} \mu$. The target thickness of the fiducial volume in liquid is $99.2 \mathrm{gm} / \mathrm{cm}^{2}$. Table $I$ is a comparison of the present and previous experiments showing $N \times$ target $g r a m s / \mathrm{cm}^{2}$, which is proportional to the total number of events at a given energy.

Table I

Present Proposal

SLAC $\left.40^{\prime \prime} B C \exp 1\right)$

FNAL, COFHMMT ${ }^{5}$ )

EMC $250 \mathrm{GeV}^{6}$ )

E665 7)
$\mathrm{N}_{\mu} \times$ Target Thickness

$3.3\left(\times 10^{10} \mathrm{gr} / \mathrm{cm}^{2}\right)$

1.4

5.1

3875

1500 
The inelastic cross section is estimated as $\sigma_{\mu}^{\text {inel }}=0.345 \mu \mathrm{b}$ for $\mathrm{Q}^{2}>1$ at $\mathrm{p}_{\mu}=$ $300 \mathrm{GeV}$ (Fig. 7). The number of events in liquid freon in the fiducial volume will be $3.3 \times 10^{10} \times 6.02 \times 10^{23} \times 0.345 \times 10^{-30}=6850$ events $\left(Q^{2} \geqslant 1\right)$. Our Wtriggering system will make a cut on $W$ and not on $Q^{2}$. For $W>4$ and $Q^{2} \geq 0$, about $40 \mathrm{~K}$ inelastic events are expected.

The inclusive strange particle production is approximately $14 \%$, which is the fraction observed in neutrino interactions in the same energy range in E745. There is an estimate of the ci production cross section, $\sigma_{\mu}{ }^{t}(c \bar{c})=9.8 * 3.3 \mathrm{nb}$ in the current fragmentation region at $p_{\mu}=250 \mathrm{GeV} / \mathrm{c}$ from the EMC results in iron ${ }^{8}$ ). The inclusive cross section for charm particle production is assumed to be $10.2 \times 2$ $\mathrm{nb}$ at $300 \mathrm{GeV} / \mathrm{c}$. The expected number of neutral strange and charm particles are

\begin{tabular}{|c|c|c|}
\hline & Produced & Observed \\
\hline$v^{o}$ & 5600 & -2800 \\
\hline 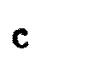 & 400 & - 150 (current fragmentation region) \\
\hline
\end{tabular}

Approximately $10 \mathrm{~K} \mu-\mathrm{Fe}$ events from the upstream chamber wall will be analyzed simultaneously with the freon events for the study of nuclear effect.

\section{$\underline{\text { References }}$}

1. J. Ballam, et. al., SLAC-PUB-1163 (1972).

2. Charm, K. Abe, et. al., Phys. Rev. D로, 1 (1986),

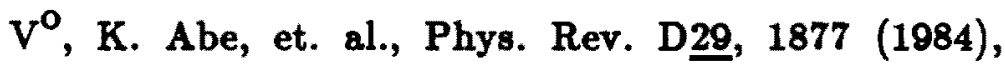

Vector meson, K. Abe, et. al., Phys. Rev. Lett. 53, 751 (1984).

3. Indirect Studies of $\mathrm{D}^{\mathrm{o}}$ and $\mathrm{V}^{\mathrm{o}}$;

EMC Collaboration CERN-EP/85-88 (1985),

EMC Collaboration CERN-EP/86-160 (1986).

There have been extensive studies of muonic charm production by dimuon or trimuon measurements. Examples are, 
J.J. Aubert, et. al., Nucl. Physics B213, 1 (1983),

J.J. Aubert, et. al., Nucl. Physics B213, 31 (1983)

M.D. Sokoloff, et. al., Fermilab-Pub-86/120-E (1986).

4. T. Kitagaki, Proc. 12th Int. Conf. on Neutrino Physics and Astrophysics, Sendai, 381 (1986).

5. M.S. Goodman, et. al., Phys. Rev. Lett. 47, 293 (1981).

6. J.J. Aubert, et. al., Phys. Lett. 105B, 322 (1981).

7. T.B.W. Kirk, E665 proposal (1985).

8. J.J. Aubert, et. al., Nucl. Phys. B213, 31 (1983). 
KINEMATICS

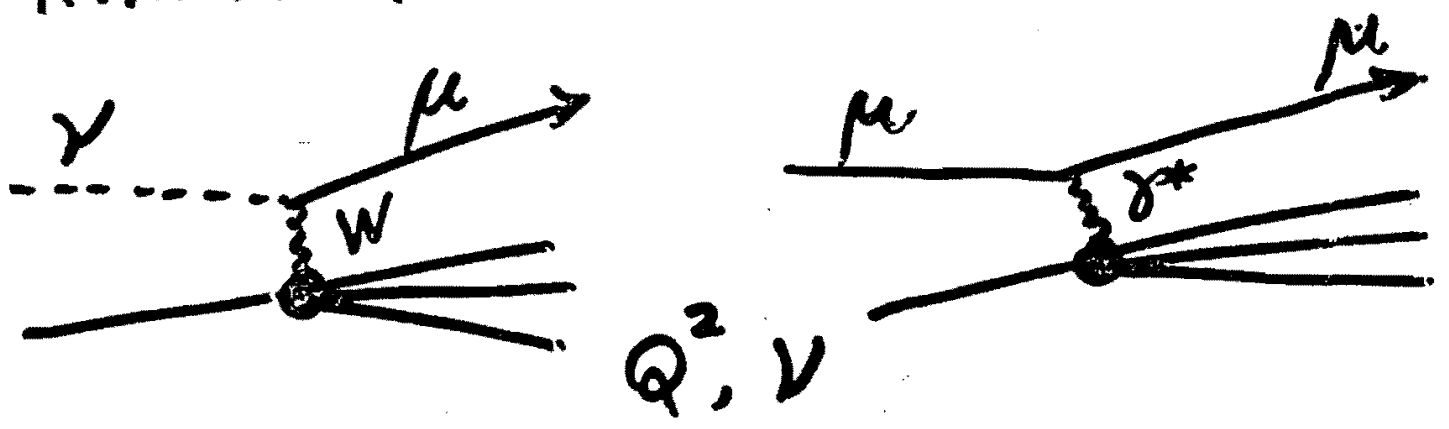

\section{INT. QUARK STATE}
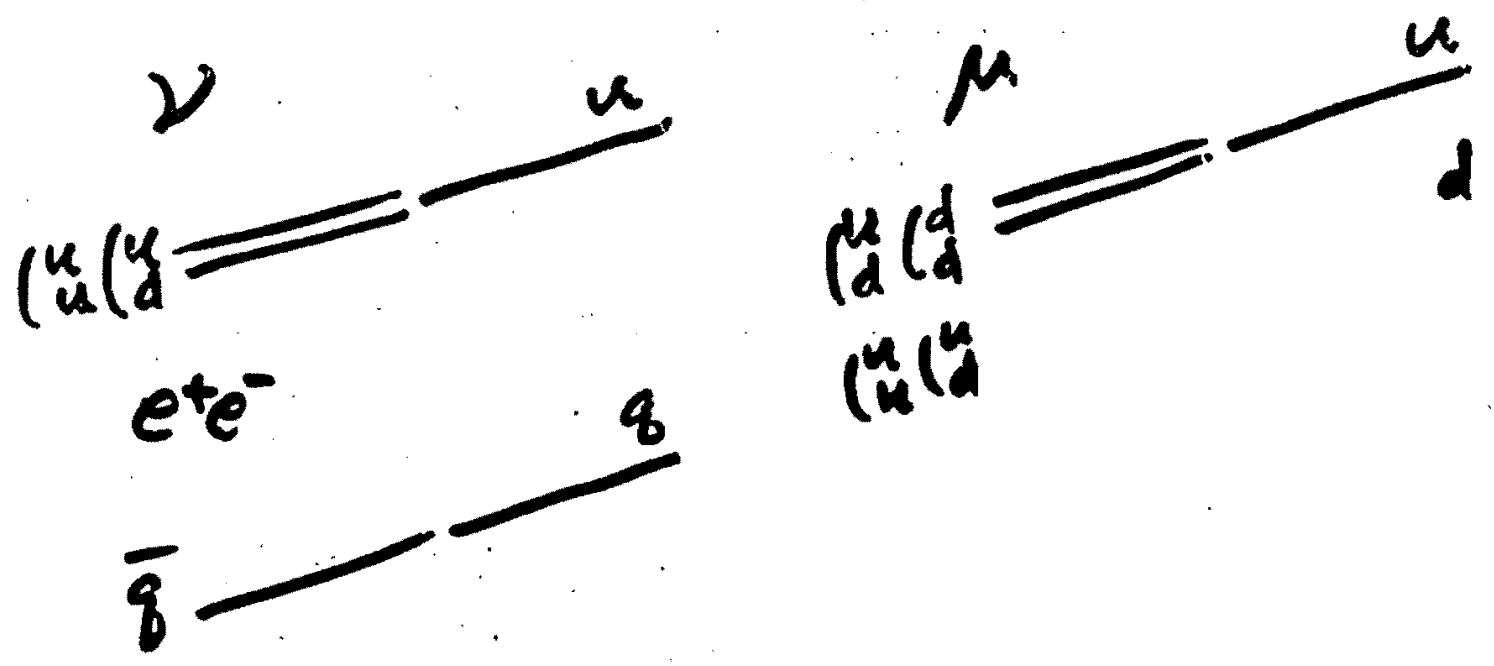

$e^{t e}$

Fig. 1 Comparison of $\nu$ and $\mu$ interactions 

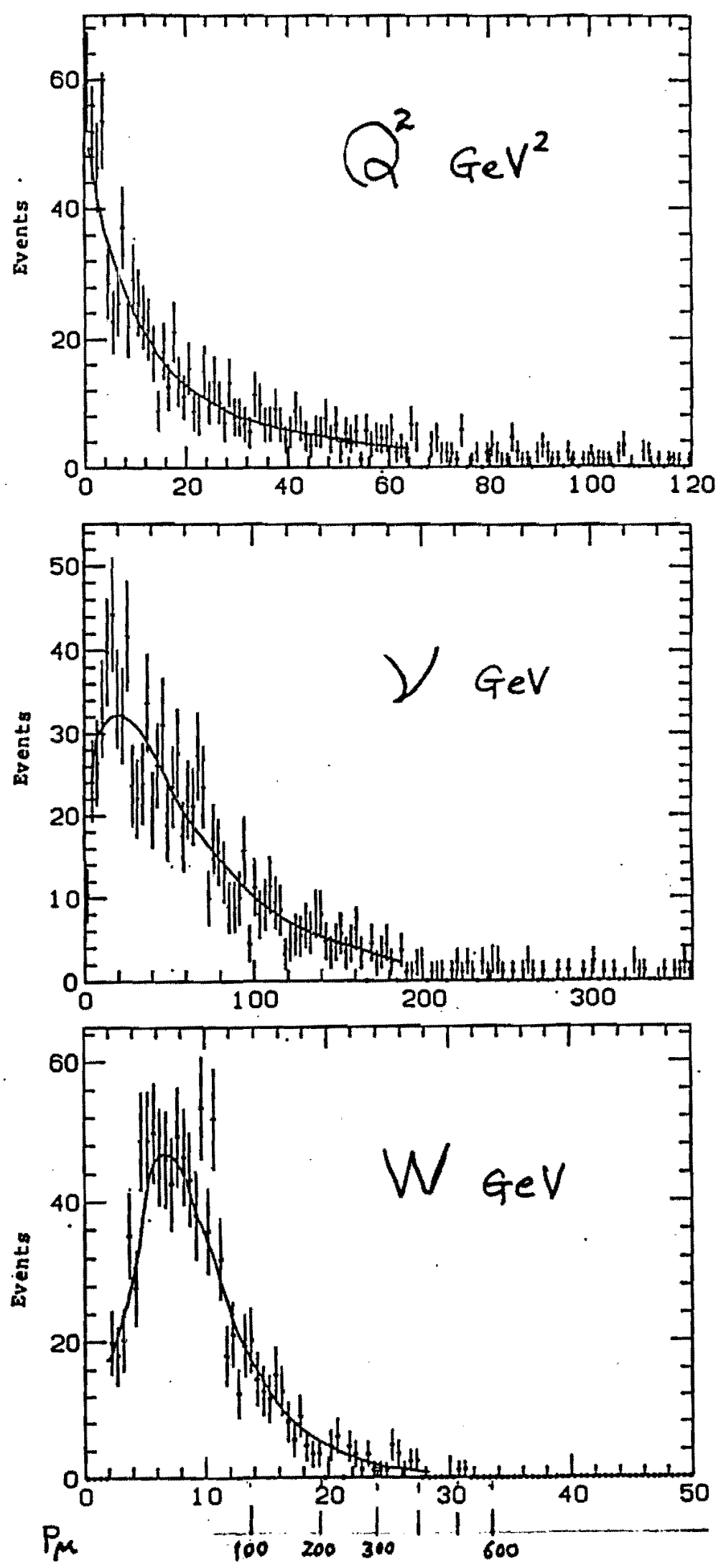

$W_{\max }$

F1g. $2 Q^{2}, v, W$ distributions.

986 vH CC events from E745 '85 run. 


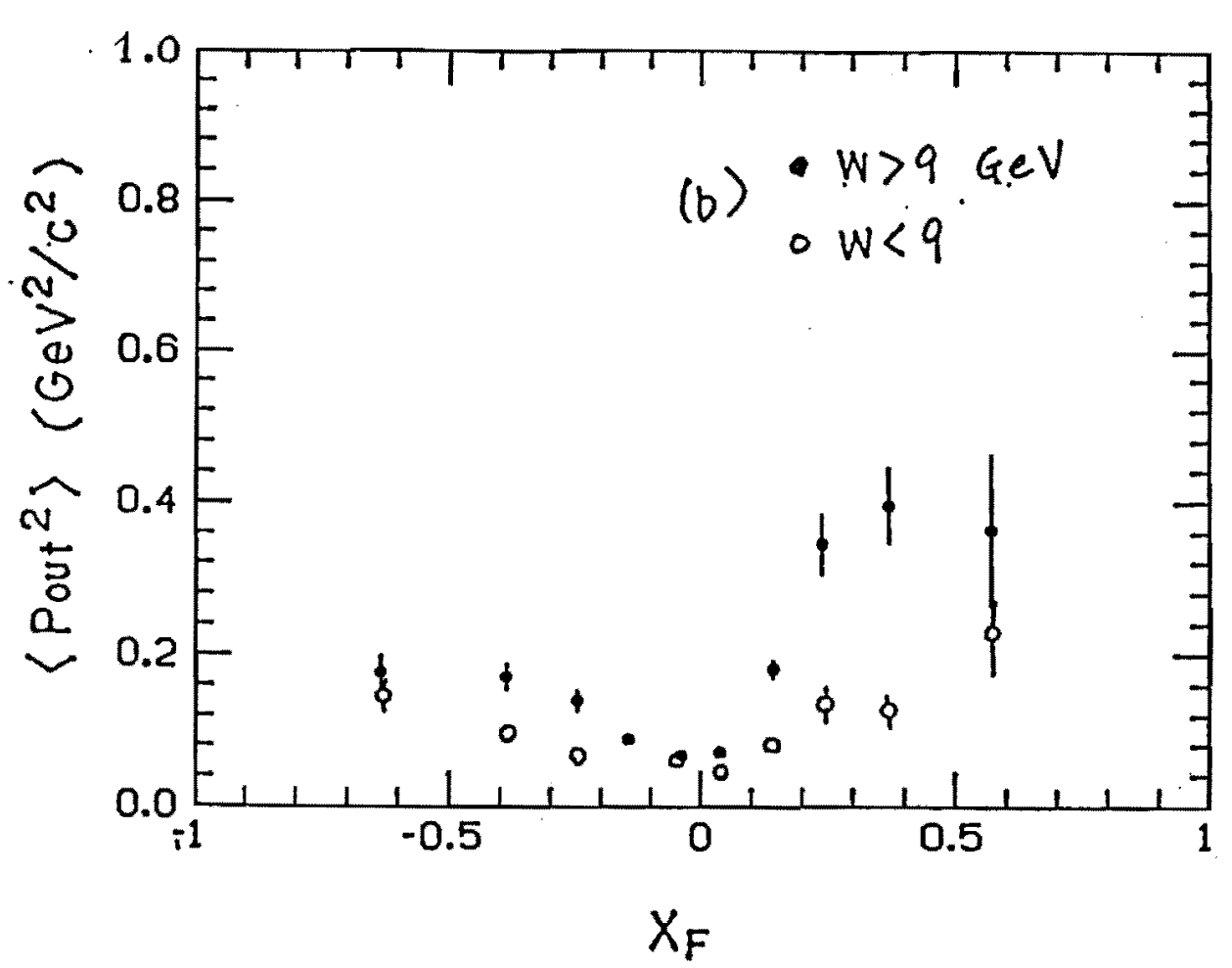

Fig. 3 (a). The averaged $P_{\text {out }}{ }^{2}$ as a function of $w$ for charged tracks.

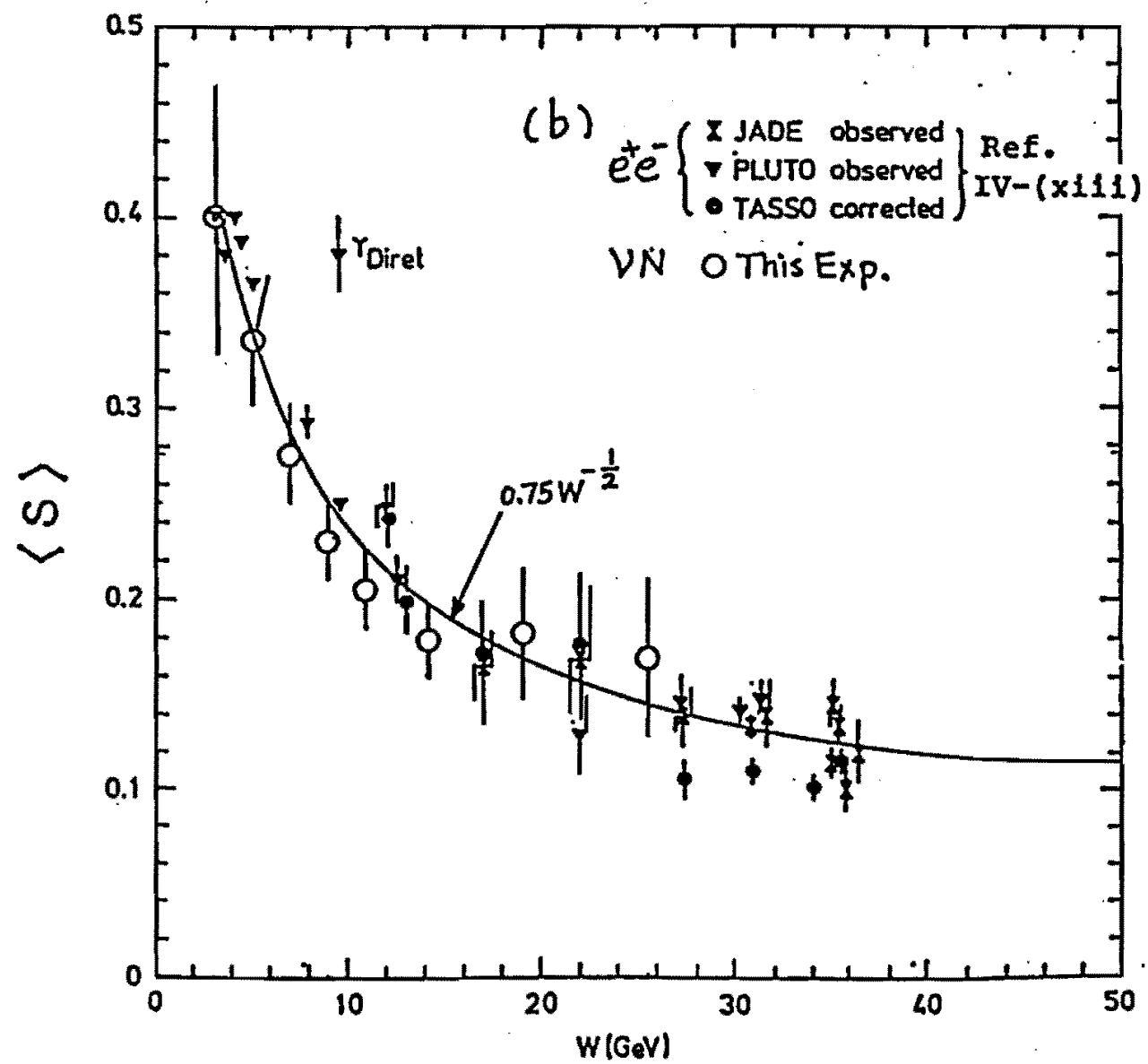

Fig. 3 (b). The averaged sphericity $s$ as a function of $w$ for $739 \mathrm{\nu} / \mathrm{CC}$ events with $n_{c h}>3$. 

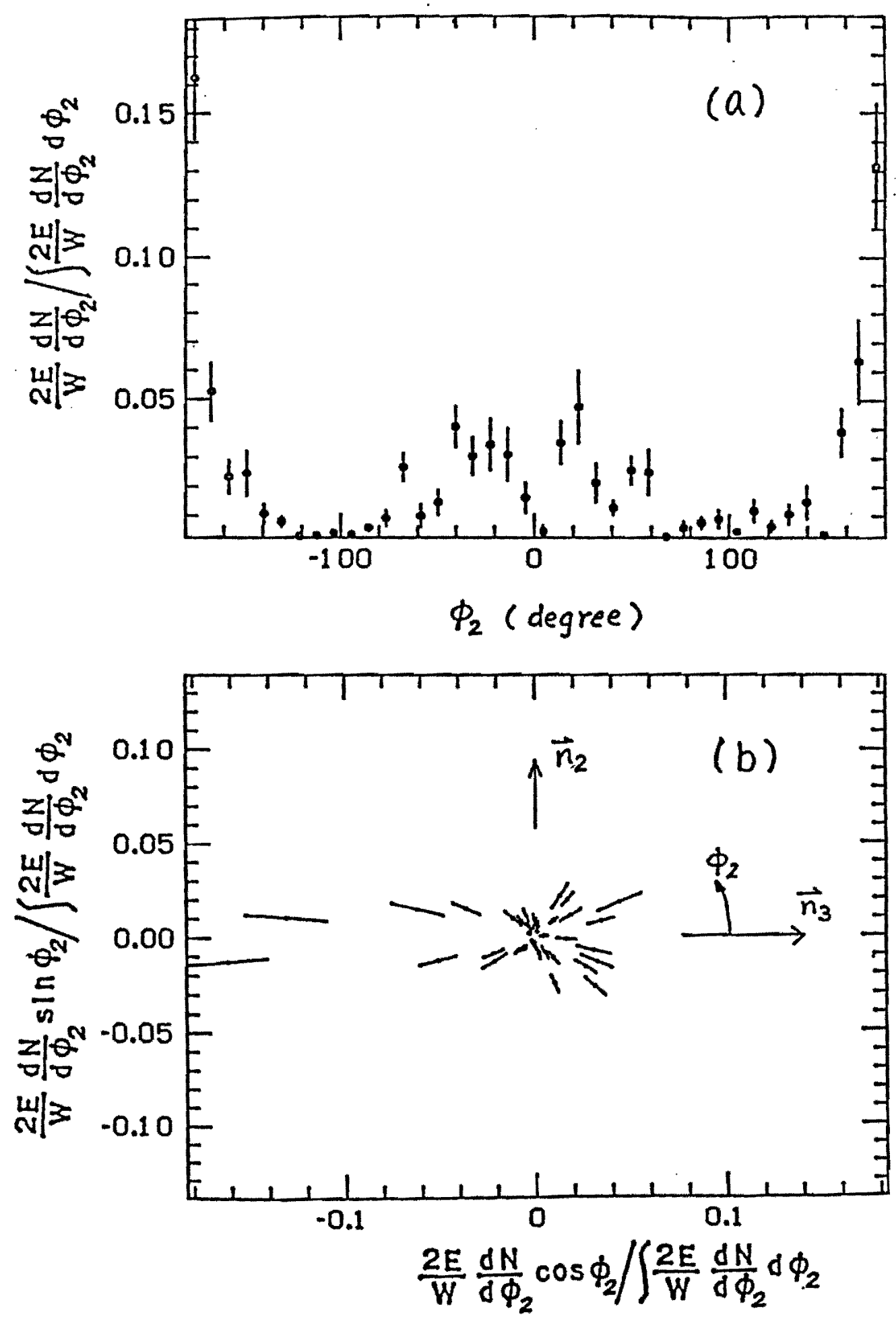

Fig. 3 (c) The angular energy flow on the $\left(\overrightarrow{n_{3}}, \overrightarrow{n_{2}}\right)$ plane for 39 events wlth $w>9 \mathrm{GeV}$, planarlty $>0.5$ and $d_{F}>2$. (d) The polar plot of data in (a). Thres jet structure is observed. 


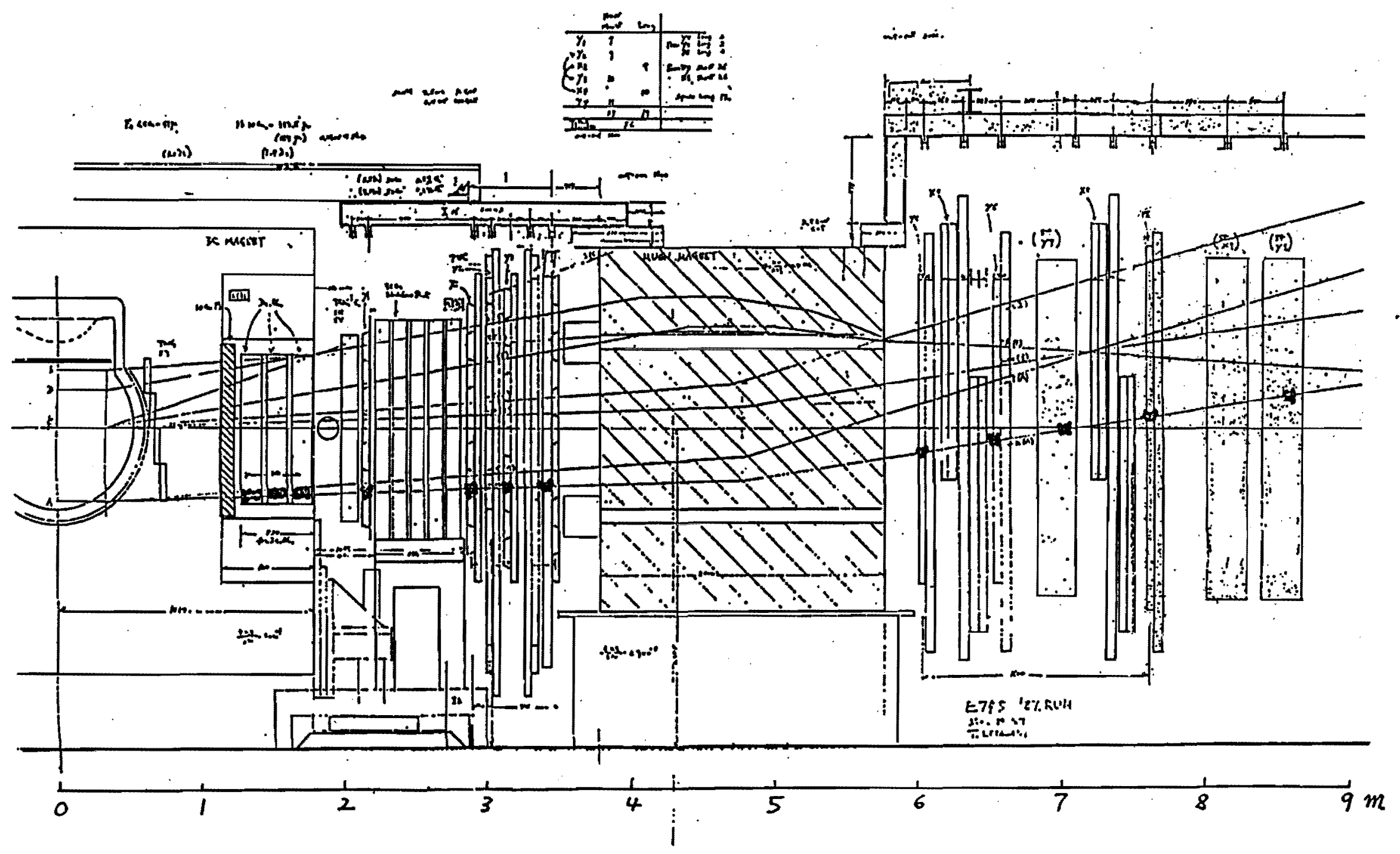

Fig. 4 Layout of E745 187 run 


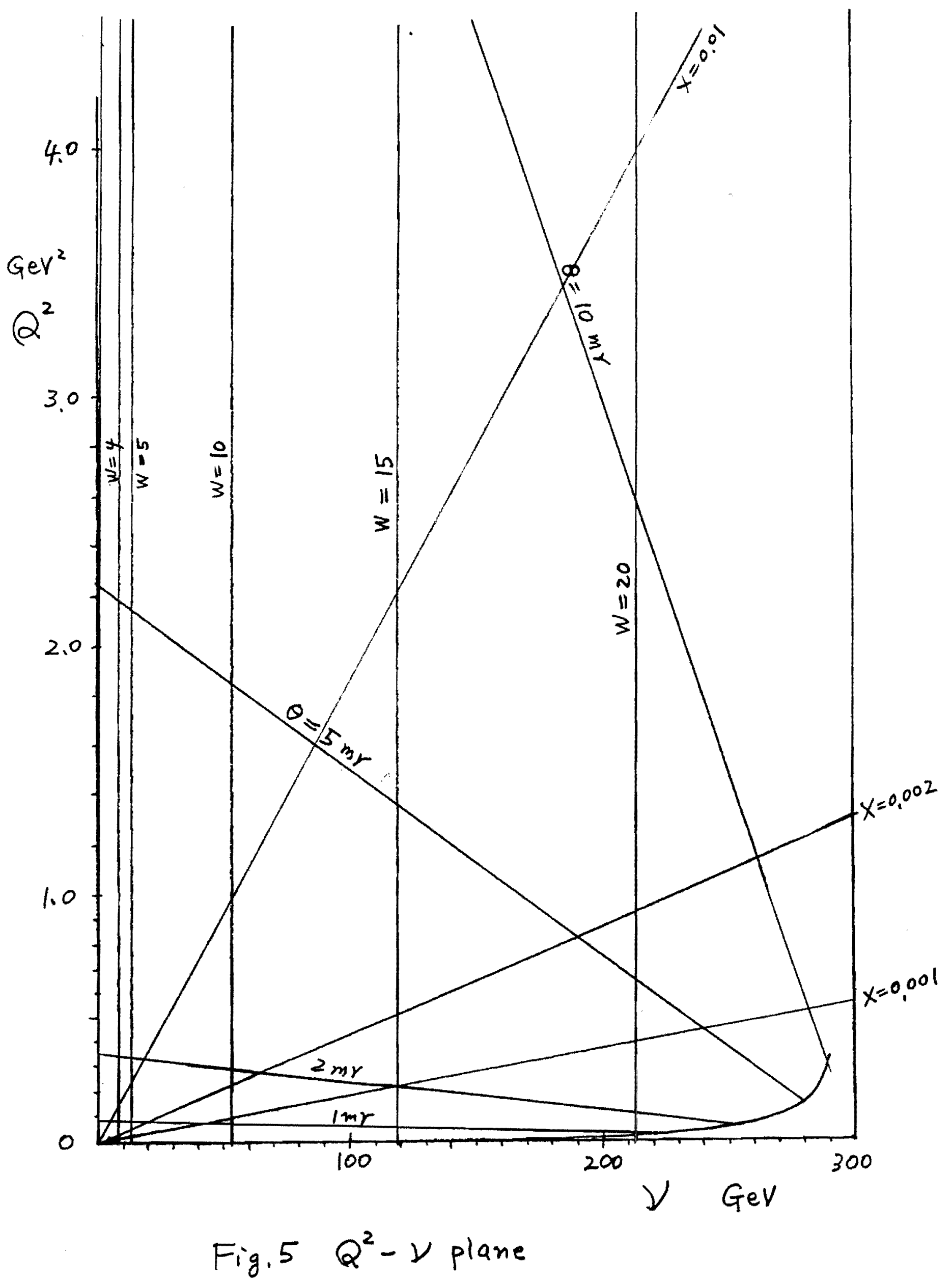




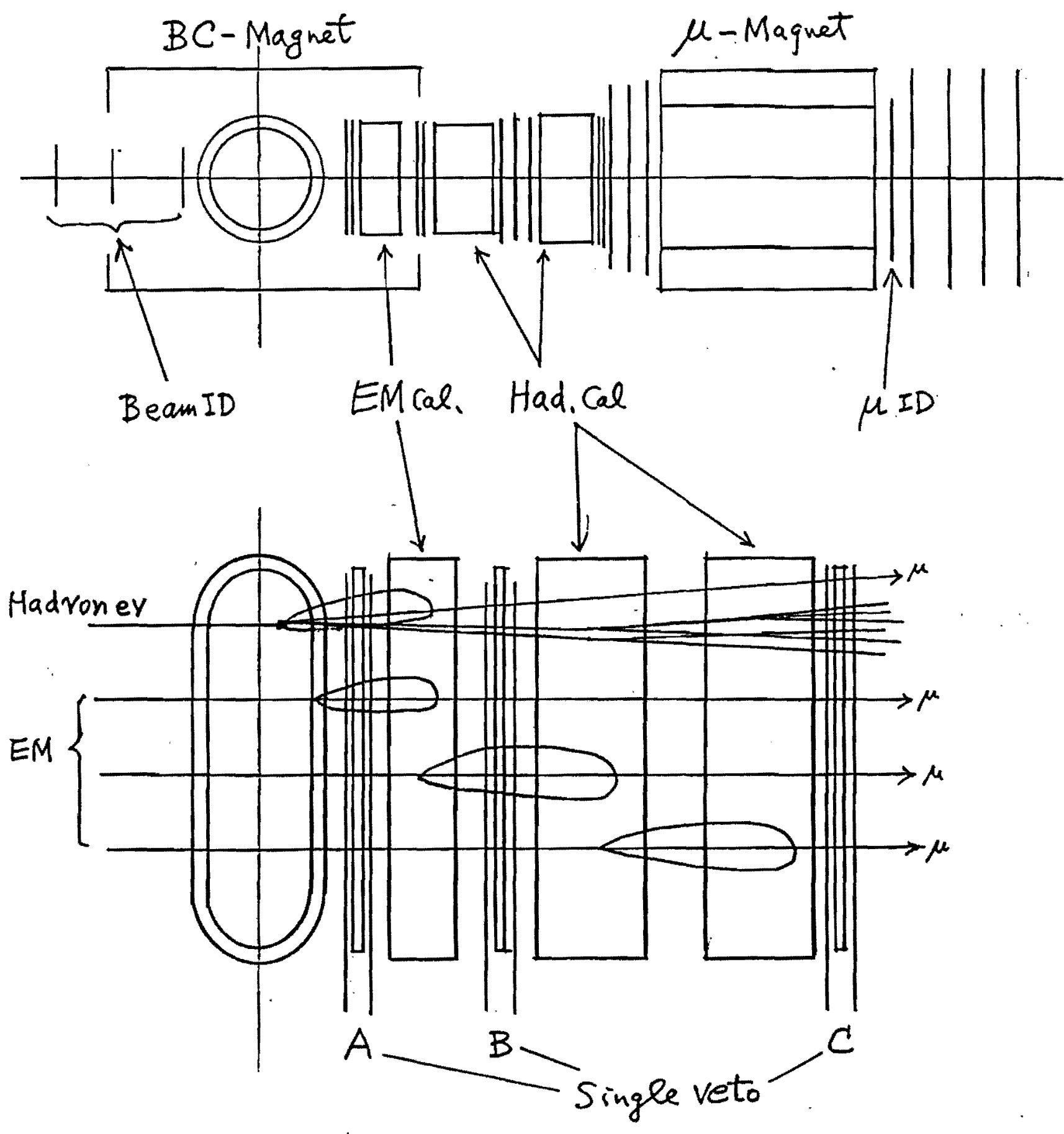

Fig. 6 W-Triggering system, Schematic.

Parameters will be optimized by a test in E745 87 run. 


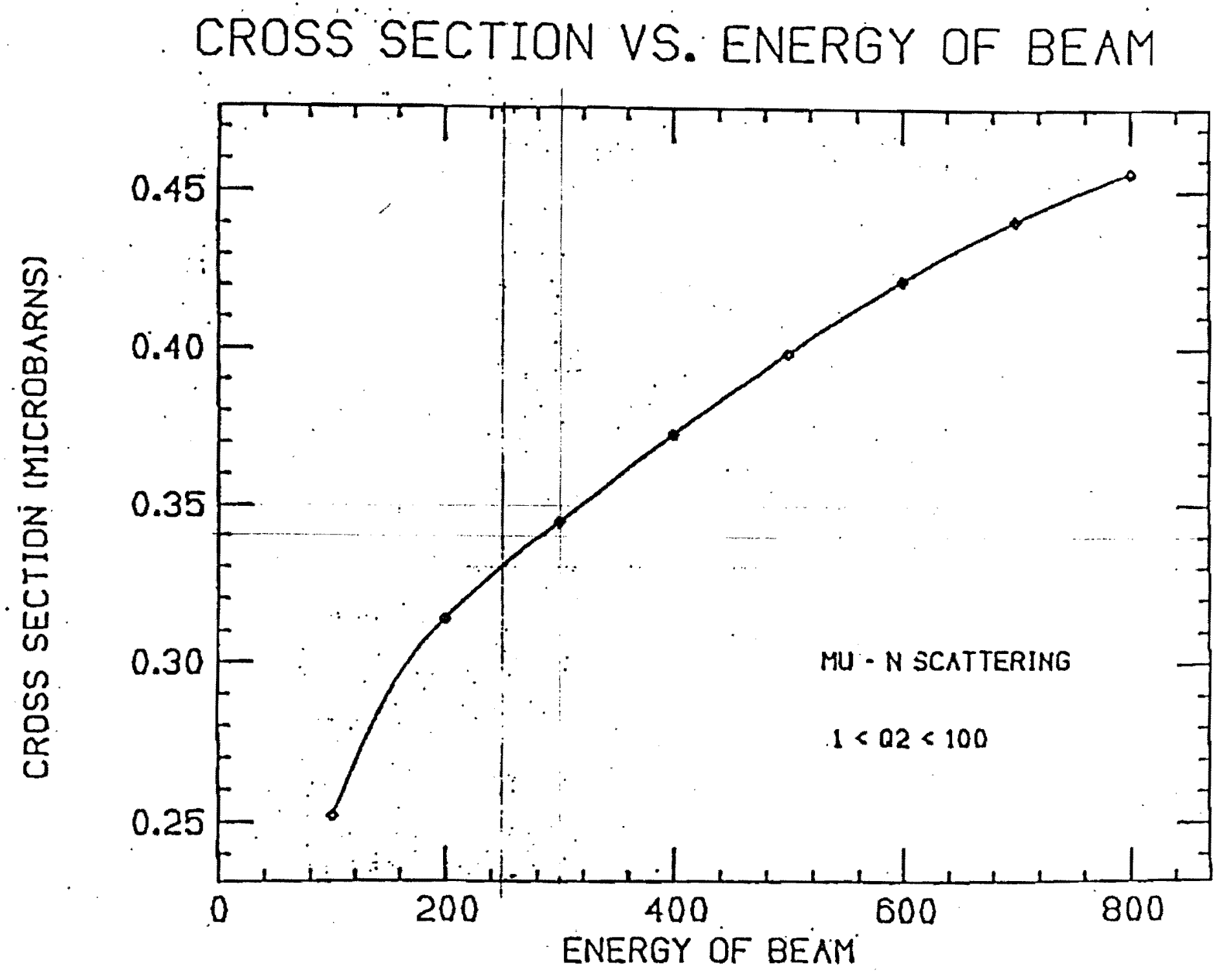

Fig. $7 \sigma\left(1<Q^{2}<100\right)$ vs $E \mu$ 